\title{
Cloroquina e hidroxicloroquina: o risco de prolongamento do intervalo QT no uso da dose recomendada para o tratamento da COVID-19
}

\author{
Chloroquine and hydroxychloroquine: the risk of QT interval prolongation when using the \\ recommended dose for the treatment of COVID-19
}

Cloroquina e hidroxicloroquina: el riesgo de prolongación del intervalo QT cuando se usa la dosis recomendada para el tratamiento de COVID-19

Mateus Camargos Silva Alves Simão ${ }^{1 *}$, Jhonson Tizzo Godoy², Antônio Mascarenhas Oliveira ${ }^{3}$, Bruno Mialarett Borja ${ }^{3}$, Felipe Santiago de Almeida ${ }^{1}$, Letícia Pereira Mendonça ${ }^{3}$, Luiza Fagundes Isolani', Adriel Gustavo Lopes ${ }^{1}$.

\section{RESUMO}

Objetivos: Avaliar através de uma revisão narrativa a relação risco-benefício do uso da cloroquina e do sulfato de hidroxicloroquina no tratamento da COVID-19, nas dosagens preconizadas pelo Ministério da Saúde, levando em consideração o possível prolongamento do intervalo QT. Revisão bibliográfica: Os estudos analisados demonstraram tanto resultados benéficos quanto nenhum resultado no combate ao SARS-Cov-19. Entretanto, explicitaram a ocorrência do prolongamento do intervalo QT em $9,1 \%$ a $25 \%$ dos pacientes, sendo este efeito adverso e suas consequências dose-dependentes. Esta repercussão adversa apresenta caráter grave, sendo potencialmente fatal. Considerações finais: Apesar de os trabalhos serem divergentes entre si, o prolongamento do intervalo QT foi frequente, evidenciando a necessidade de um monitoramento eletrocardiográfico subsequente dos pacientes. Além disso, é necessário produzir maior quantidade de estudos para uma conclusão segura acerca do uso seguro da terapia avaliada, visto que os riscos do uso da cloroquina e do sulfato de hidroxicloroquina devem ser superados pelos benefícios do tratamento, que ainda apresenta sua eficácia em discussão.

Palavras-chave: Hidroxicloroquina, Cloroquina, Coronavírus, Eletrocardiografia.

\begin{abstract}
Objective: Evaluate through a narrative review the risk-benefit ratio of the use of chloroquine and hydroxychloroquine sulfate in the treatment of COVID-19, in the dosages recommended by the Ministry of Health, taking into account the possible prolongation of the QT interval. Literature review: The analyzed studies showed both beneficial results and no results in combating SARS-Cov-19. However, they demonstrated the occurrence of QT interval prolongation in $9.1 \%$ to $25 \%$ of patients, with this adverse effect and its consequences being dose-dependents. This adverse repercussion is serious and potentially fatal. Final considerations: Although the studies are divergent, QT interval prolongation was frequent, showing the need for subsequent electrocardiographic monitoring of patients. In addition, it is necessary to produce a greater number of studies for a safe conclusion about the safe use of the evaluated therapy, since the risks of using chloroquine and hydroxychloroquine sulfate must be outweighed by the treatment benefits, and these benefits are still in analysis.
\end{abstract}

Keywords: Hydroxychloroquine, Chloroquine, Coronavirus, Electrocardiography.

\footnotetext{
${ }_{1}^{1}$ Pontifícia Universidade Católica de Minas Gerais (PUC-MG), Betim - MG.

*E-mail: camargosmateus@gmail.com

2 Santa Casa de Misericórdia de Belo Horizonte, Belo Horizonte - MG.

${ }^{3}$ Faculdade de Minas (FAMINAS), Belo Horizonte - MG.
} 


\section{RESUMEN}

Objetivos: Evaluar mediante una revisión narrativa la relación riesgo-beneficio del uso de cloroquina y sulfato de hidroxicloroquina en el tratamiento de COVID-19, en las dosis recomendadas por el Ministerio de Salud, teniendo en cuenta la posible prolongación del intervalo QT. Revisión de la literatura: Los estudios analizados mostraron resultados beneficiosos y ningún resultado en la lucha contra el SARS-Cov-19. Sin embargo, demostraron la aparición de la prolongación del intervalo QT en el 9,1\% al 25\% de los pacientes, con este efecto adverso y sus consecuencias dependientes de la dosis. Esta repercusión adversa es grave y potencialmente mortal. Consideraciones finales: Aunque los estudios son divergentes, la prolongación del intervalo QT fue frecuente, lo que demuestra la necesidad de una monitorización electrocardiográfica posterior de los pacientes. Además, es necesario producir un mayor número de estudios para llegar a una conclusión segura sobre el uso seguro de la terapia evaluada, ya que los riesgos del uso de cloroquina y sulfato de hidroxicloroquina deben ser superados por los beneficios del tratamiento, y estos beneficios aún están en análisis.

Palabras clave: Hidroxicloroquina, Cloroquina, Coronavirus, Electrocardiografía.

\section{INTRODUÇÃO}

No fim do ano de 2019 o surgimento de um novo coronavírus, Síndrome Respiratória Aguda Grave Coronavírus 2 (SARS-Cov-2), desencadeou um surto de uma nova doença, denominada Doença pelo Coronavírus 2019 (COVID-19), iniciado em Wuhan, na China. Esta enfermidade, a partir de seu alto poder de transmissibilidade e rápida disseminação, atingiu proporções mundiais, sendo classificada como pandemia pela Organização Mundial da Saúde (OMS) no dia 12 de março de 2020. Responsável por incidência e número de óbitos crescentes, a doença é causa de ameaça global aos âmbitos econômico, social e de saúde pública (GAUTRET P, et al., 2020).

Levando em conta a ausência de um medicamento específico para o tratamento da doença, vários testes clínicos foram realizados para a determinação de protocolos terapêuticos com outras drogas, dentre os quais a cloroquina (CQ) e seu análogo, o sulfato de hidroxicloroquina (HCQ), mostraram atividade inibitória in vitro semelhante na infecção pelo SARS-Cov-2. Com adição de um grupo hidroxila, a HCQ apresentou menor toxicidade em relação à $C Q$.

Em uma análise de 16 testes clínicos chineses, mais de 100 pacientes demonstraram superioridade do sulfato de hidroxicloroquina em relação à sua precursora no que diz respeito ao tratamento da COVID-19. Com provável atuação nas fases de entrada e pós-entrada do vírus na célula, os fármacos agem através de efeitos no pH do endossoma, resultando na subglicosilação da enzima conversora de Angiotensina 2, necessária para a entrada viral (LIU, et al., 2020; TOURET F e LAMBALLERIE X, 2020; GIUDICESSI JR, et al., 2020).

O Ministério da Saúde (MS) publicou, no dia 17 de abril de 2020, a terceira versão das Diretrizes para o Diagnóstico e Tratamento da COVID-19. Segundo o documento, até o momento, não existem evidências robustas de alta qualidade que possibilitem a indicação de uma terapia farmacológica específica para a COVID-19. Contudo, seguindo as poucas evidências identificadas, por meio da Nota Informativa № 6/2020 do Departamento de Assistência Farmacêutica e Insumos Estratégicos (DAF/SCTIE/MS), o órgão admitiu o uso da cloroquina e do sulfato de hidroxicloroquina em casos confirmados da doença. O emprego dos fármacos deve ser feito a critério médico, como terapia adjuvante no tratamento de formas graves da COVID-19, em pacientes hospitalizados, sem que outras medidas de suporte sejam preteridas (MS, 2020).

Ainda de acordo com a Nota Informativa, no primeiro dia do tratamento, a cloroquina deve ser oferecida em dose de ataque de $900 \mathrm{mg}$ diárias, dividida em duas doses de $450 \mathrm{mg}$ ao dia. Nos próximos 4 dias deve-se administrar 450mg diárias em apenas uma dose. Já o sulfato de hidroxicloroquina apresenta dose de ataque de $800 \mathrm{mg}$ diárias, dividida em duas doses de $400 \mathrm{mg}$ ao dia, esquema que também deve ser realizado no primeiro dia do tratamento. Nos próximos 4 dias deve-se administrar um comprimido de $400 \mathrm{mg}$ ao dia. Em ambos os casos, totaliza-se 5 dias da terapia (MS, 2020). 
Utilizados no tratamento da artrite reumatóide, artrite reumatóide juvenil, lúpus eritematoso sistêmico e discóide, condições dermatológicas provocadas ou agravadas pela luz do sol e malária, cloroquina e sulfato de hidroxicloroquina possuem entre possíveis efeitos o prolongamento do intervalo QT. Este prolongamento constitui um aumento do tempo da repolarização ventricular, resultante de alterações dos fluxos de potássio no potencial de ação correspondente a partir do bloqueio de canais Kv11.1, codificados pelo gene humano relacionado ao éter-a-go-go ( $h E R G$ ).

O intervalo QT é prolongado quando tem uma duração maior que 480 milissegundos (ms) em pacientes do sexo feminino pós-puberdade, maior que $470 \mathrm{~ms}$ nos do sexo masculino pós-puberdade e $460 \mathrm{~ms}$ em pacientes de ambos os sexos pré-puberdade. Esse distúrbio de condução elétrica cardíaca tem associação íntima com as taquiarritmias ventriculares, como a Torsades de Pointes (TdP) ou taquicardia polimórfica ventricular. Evoluções possíveis são síncope, fibrilação ventricular e morte súbita (LAKKIREDDY DR, et al., 2020; GIUDICESSI JR, et al., 2020; PRUTKIN JM, et al., 2020).

Com a inserção da CQ e HCQ em diversos protocolos nacionais para o tratamento da COVID-19 o estabelecimento da relação de risco-benefício de tais drogas é imprescindível, sendo a eficácia destas ainda debatida. Dentre seus possíveis efeitos colaterais o prolongamento do intervalo QT se apresenta como uma consequência grave, levando à necessidade de uma administração cautelosa destes fármacos no combate ao SARS-Cov-2. Considerando este contexto, este projeto tem como objetivo avaliar, através de uma revisão narrativa, o benefício desta abordagem terapêutica, nas dosagens preconizadas pelo Ministério da Saúde.

\section{REVISÃO BIBLIOGRÁFICA}

\section{Estudos favoráveis quanto aos efeitos da Cloroquina e Hidroxicloroquina}

Um estudo francês demonstrou que o uso diário de $600 \mathrm{mg}$ de HCQ ( 3 doses de $200 \mathrm{mg}$ ao dia), durante 10 dias, apresentou $70 \%$ de cura viral ao sexto dia de sua administração. Os participantes deveriam ter idade superior a 12 anos, sendo excluídos aqueles que apresentassem prolongamento do intervalo QT anterior ao tratamento. Este projeto mostrou associação significativa com a redução da carga viral e consequente cura da infecção pelo SARS-Cov-2 pela HCQ, na análise de 8 pacientes de uma amostra total de 36 (GAUTRET P, et al., 2020).

Um estudo chinês demonstrou melhora do quadro clínico de pneumonia na COVID-19 em 80,6\% dos pacientes, estando estes em uso do sulfato de hidroxicloroquina por 5 dias, na dose diária de $400 \mathrm{mg}$. Foi relatada melhora do quadro febril e da tosse, além da redução nos achados em exames de imagem do tórax. Em comparação, tal evolução benéfica foi percebida em apenas $54,8 \%$ dos pacientes que não realizaram tratamento com $\mathrm{HCQ}$, havendo ainda progressão da doença em 4 casos deste último grupo. $\mathrm{O}$ benefício do uso do sulfato de hidroxicloroquina no tratamento da doença foi expressivo na amostra de 62 pacientes abordada pelo estudo (CHEN Z, et al., 2020).

Um segundo estudo chinês utilizou amostra de 22 pacientes. Destes, 10 participantes fizeram uso de $500 \mathrm{mg}$ de cloroquina, duas vezes ao dia, durante dez dias. Os demais 12 participantes, membros do grupo controle, foram tratados com outra droga antirretroviral. O grupo abordado com administração de $\mathrm{CQ}$ apresentou exames negativos para SARS-Cov-2 de todos os seus integrantes após 13 dias do início da terapia. O grupo teste não apresentou exames negativos para todos os seus integrantes nem mesmo após 14 dias (HUANG M, et al., 2020).

\section{Estudos inconclusivos quanto aos efeitos da Cloroquina e Hidroxicloroquina}

Um estudo chinês, prospectivo, randomizado e aberto, dividiu 30 pacientes diagnosticados com COVID19 em dois grupos de 15 pessoas. O grupo teste recebeu tratamento com cloroquina, na dose de $400 \mathrm{mg}$, duas vezes ao dia, durante 5 dias. O grupo controle realizou tratamento convencional, que inclui repouso no leito, oxigenoterapia e suporte sintomático. Ao final de 2 semanas não foi detectada diferença significativa na evolução dos dois grupos, não sendo percebido benefício expressivo com o tratamento com a cloroquina, inclusive tendo o grupo controle demandando maior tempo para normalização da temperatura corporal. De acordo com este estudo, para uma determinação eficaz dos benefícios do tratamento com a $\mathrm{CQ}$, seria necessária uma amostra mínima de 784 pacientes (CHEN J, et al., 2020). 
Um estudo americano analisou a relação entre o uso da Hidroxicloroquina, Cloroquina, Azitromicina e Amoxacilina com os desfechos "morte" e "Torsades de Pointes/Prolongamento do intervalo QT". Segundo os dados coletados, o uso da HCQ e CQ de forma isolada não apresenta potencial de segurança para o desenvolvimento de Torsades de Pointes/Prolongamento do intervalo QT e para morte. Foram analisadas mais de 13 milhões de notificações entre pacientes em uso de HCQ e CQ, Azitromicina e HCQ/CQ, ou Azitromicina ou Amoxacilina. Os dados foram coletados de 1964 até 2019.

É válido ressaltar que nenhum dos pacientes utilizou qualquer das medicações como alternativa para o tratamento de SARS-Cov-2. Dessa forma, é necessária a análise destes mesmos desfechos para o tratamento da respectiva doença (SARAYANI A, et al., 2020).

Um segundo estudo francês, retrospectivo, avaliou prontuários eletrônicos de pacientes internados em centros terciários de saúde com pneumonia por COVID-19, sendo a amostra composta por 181 pacientes, com idade entre 18 e 80 anos, com exame confirmatório para a infecção e com necessidade de oxigenoterapia, divididos em grupos caso-controle de acordo com a terapêutica em uso. Destes, 84 realizaram uso diário de $600 \mathrm{mg}$ de $\mathrm{HCQ}$ nos dois primeiros dias de hospitalização, ao passo que 97 pacientes não foram tratados com esta droga. Os pacientes que estavam em uso de HCQ permaneceram até sua morte, cura da infecção ou até 7 dias após alta hospitalar.

De acordo com a pesquisa, os pacientes tratados com a droga tiveram uma menor probabilidade de transferência para a unidade de terapia intensiva e de mortalidade. Segundo os autores, 9 pacientes em uso do sulfato de hidroxicloroquina tiveram alterações eletrocardiográficas, em média no quarto dia de tratamento, requerendo a descontinuação da terapêutica. Dentre esses indivíduos, 7 tiveram um intervalo QT corrigido (QTc) com duração maior que $60 \mathrm{~ms}$ do valor da normalidade do estudo e 1 teve um prolongamento grave de QT com QTc, de valor maior que $500 \mathrm{~ms}$.

A inconclusividade do estudo em questão se dá pela existência concomitante de efeitos favoráveis e desfavoráveis ao da $C Q$ e $H C Q$, representados, respectivamente, pela redução do risco de internação em unidade de terapia intensiva e o aumento do risco de prolongamento do intervalo QT (MAHEVAS M, et al., 2020).

Geleris J, et al. (2020) adotou a estratégia de análise de 1376 pacientes, entre os quais $811(58,9 \%)$ fizeram uso de $600 \mathrm{mg}$ de Hidroxicloroquina no primeiro dia de tratamento e $400 \mathrm{mg}$ nos 4 dias seguintes. Os demais 565 pacientes não foram tratados com este fármaco. Dentre os pacientes medicados, 262 apresentaram necessidade de intubação ou foram ao óbito. Já dentre os 565 pacientes não medicados, 84 tiveram algum de tais desfechos descritos.

As informações do estudo, no entanto, não demonstram um aumento expressivo no risco para estas complicações relacionadas ao uso da Hidroxicloroquina, mas ressalta a necessidade de mais estudos para a verificação da eficácia desta terapia.

\section{Estudos desfavoráveis quanto aos efeitos da Cloroquina e Hidroxicloroquina}

Um estudo brasileiro apresenta resultados de um ensaio clínico fase Ilb, duplo-cego e randomizado sobre o uso da cloroquina em duas doses diferentes, empregada como terapia em 81 pacientes hospitalizados com síndrome respiratória grave, infectados pelo SARS-Cov-2. Os grupos foram submetidos ao tratamento com a $C Q$ em doses diferentes, sendo o primeiro na dose de $600 \mathrm{mg}$, duas vezes ao dia, durante 10 dias (total de 12g) e o segundo na dose de $450 \mathrm{mg}$, duas vezes ao dia, por 3 dias (total de 2,7g). Todos os pacientes usavam ainda ceftriaxona intravenosa e azitromicina, além de oseltamivir quando ocorria suspeita de infecção por influenza.

Segundo o estudo, a dosagem mais elevada de cloroquina gera uma tendência maior, em relação à dosagem mais baixa, para o prolongamento do intervalo QT acima de 500 milissegundos (25\%) e para a letalidade (17\%). Dentre os 440 pacientes da amostra, 81 apresentaram complicações cardíacas com o uso da CQ. Além disso, no grupo de alta dosagem dois pacientes evoluíram com taquicardia ventricular antes da morte, um quadro de arritmia favorecido pelo prolongamento de QT (BORBA MGS, et al., 2020). 
Um terceiro estudo francês analisou as repercussões cardíacas consequentes do uso crônico de cloroquina ou sulfato de hidroxicloroquina por 127 pacientes. A média de tempo de uso dos fármacos pelos participantes foi avaliada em 7 anos, sendo as dosagens variáveis. De acordo com esta análise, o efeito adverso mais reportado diz respeito aos distúrbios de condução, afetando $85 \%$ dos pacientes abordados (CHATRE C, et al., 2020).

\section{Discussão sobre os estudos}

De acordo com uma análise, cloroquina e hidroxicloroquina interferem de forma semelhante na repolarização ventricular, prolongam o intervalo QT e aumentam o risco de Torsades de Pointes. Estes efeitos adversos apresentam-se como dose dependentes, situação representada por estudos envolvendo voluntários. Nestes trabalhos foram citados aumento do intervalo QT de $6.1 \mathrm{~ms}$ depois da administração de $600 \mathrm{mg}$ de $\mathrm{CQ}$, além de prolongamento de $28 \mathrm{~ms}$ depois da administração de $1200 \mathrm{mg}$ da mesma droga (JUURLINK DN, 2020).

Os resultados obtidos permitem uma análise ampla sobre o tema, porém pouco aprofundada. Por seu caráter recente, a infecção pelo SARS-Cov-2 e seu tratamento ainda não são bem elucidados, especialmente pelo pequeno número de estudos concluídos acerca da temática e a pequena representatividade das amostras nos que foram finalizados. Chen J, et al. (2020), a título de exemplo, utilizou amostra de 30 pacientes, mas avaliou que um valor mínimo para que sua amostra fosse considerada representativa seria de 784 participantes. Dessa maneira, demonstra-se a necessidade por mais estudos que comprovem a efetividade do tratamento proposto, apresentando resultados com maior validade a partir de amostras mais numerosas.

Gautret P, et al. (2020) demonstrou que o uso da HCQ apresentou significativos benefícios no tratamento da Doença pelo Coronavírus 2019, expondo efeito redutor da carga viral e atenuando seu quadro clínico e complicações. Chen Z, et al. (2020) apresentou resultados semelhantes em seu estudo. Apesar disso, as amostras utilizadas por ambos os trabalhos foram pouco representativas, computadas em 36 e 62 pacientes, respectivamente.

Chen J, et al. (2020), adjacente aos estudos que mostraram benefício na referida terapêutica, alertou para a não constatação de diferenças significativas no combate ao SARS-Cov-2 em pacientes que fizeram uso da $\mathrm{HCQ}$ e os que fizeram tratamento convencional. Deve-se levar em conta o valor reduzido da amostra utilizada neste estudo, avaliada em 30 pacientes.

Huang M, et al. (2020) demonstrou, a partir de seu estudo, a eficácia da cloroquina no combate ao SARS-Cov-2, inclusive com maior eficiência em relação à demais drogas antivirais testadas. Desta forma, tais resultados apresentam mais uma evidência da utilidade do medicamento no tratamento da COVID-19. No entanto torna-se válido ressaltar que este estudo também carece de uma amostra representativa, tendo sido utilizados apenas 22 pacientes para o desenvolvimento da pesquisa.

$\mathrm{Na}$ maioria dos estudos foi constatada uma relação entre o prolongamento do intervalo QT e o uso da CQ ou HCQ. Borba MGS, et al. (2020) relatou intervalo QT maior que $500 \mathrm{~ms}$ em $25 \%$ dos pacientes em uso da maior dosagem de $C Q$, tendo este mesmo grupo apresentado maior incidência para taquicardia ventricular, arritmia e consequente óbito. Mahevas M, et al. (2020), por sua vez, constatou incidência do prolongamento do intervalo QT de 10,7\%. Chatre C, et al. (2020) observou que entre 127 pacientes em uso de cloroquina ou sulfato de hidroxicloroquina, $85 \%$ apresentaram distúrbios de condução cardíaca como efeito adverso da terapia, sendo esta a consequência indesejada mais frequente em meio às alterações cardíacas citadas.

Em referência ao risco-benefício da terapia com cloroquina ou sulfato de hidroxicloroquina, constatou-se que ambos os tratamentos apresentam benefícios. Borba MGS, et al. (2020) demonstrou que o aumento da dose de cloroquina é proporcional ao aumento do risco das complicações do prolongamento do intervalo QT. No que diz respeito à $H C Q$, o conjunto de dados acerca da dose-dependência em seus efeitos adversos é insuficiente. Conclusões acerca das consequências terapêuticas de ambas as drogas não podem ser realizadas por inferências. 
Prutkin JM, et al. (2020) demonstrou que a HCQ apresenta menor risco de desenvolver Torsades de Pointes quando usada para o tratamento de artrite reumatoide, lúpus eritematosos e malária quando comparada à $\mathrm{CQ}$. Entretanto, ainda que tais deduções não sejam possíveis, considerando a posição da $\mathrm{HCQ}$ de análoga da $C Q$, apresentando propriedades farmacológicas semelhantes, precauções quanto a doses mais altas do sulfato de hidroxicloroquina devem ser tomadas.

Em suma, o benefício oferecido pelo uso do sulfato de hidroxicloroquina no tratamento da COVID-19 tem caráter incerto, visto que os trabalhos realizados até o momento se mostraram pouco representativos. Em relação aos riscos, o prolongamento do intervalo QT mostrou-se como efeito adverso recorrente nesta proposta terapêutica.

\section{Alternativas de manejo clínico}

Os pacientes a serem submetidos ao tratamento da COVID-19 através do uso da HCQ e CQ devem ser avaliados quanto ao risco de desenvolver prolongamento do intervalo QT, considerando o potencial de fatalidade gerado por este distúrbio de condução ventricular. Kapoor A, et al. (2020) explica que tais pacientes podem ser classificados como baixo risco (intervalo QT normal), risco moderado (intervalo QT de até $500 \mathrm{~ms}$ ) ou de alto risco (intervalo QT maior que $500 \mathrm{~ms}$ ). O Ministério da Saúde (2020), por sua vez, preconiza que todo paciente a ser submetido ao tratamento de SARS-Cov-2, tanto com CQ, quanto com $\mathrm{HCQ}$ deverá, antes da terapia, realizar eletrocardiograma. Em seguimento, os pacientes devem ser submetidos à realização do ECG também nos dias subsequentes, monitorando o intervalo QT durante todo o período de internação.

Prutkin JM, et al. (2020) demonstrou que a melhor maneira para monitorar um eventual prolongamento do intervalo QT em pacientes em uso de $C Q$ ou $H C Q$ é feita a partir de eletrocardiogramas de 12 derivações. Segundo o autor, intervalos QTc menores que $460 \mathrm{~ms}$ em pré-púberes, menores que $470 \mathrm{~ms}$ em homens pós-púberes e menores que $480 \mathrm{~ms}$ em mulheres pós-púberes caracterizam pacientes com baixo risco para prolongamentos significativos deste intervalo ou taquicardia ventricular polimórfica. Pacientes de alto risco para as mesmas condições apresentariam intervalo QTc maior ou igual a $500 \mathrm{ms,}$ juntamente com um QRS maior ou igual a $120 \mathrm{~ms}$.

Segundo a American Heart Association o uso de uma droga sabidamente capaz de desenvolver arritmia do tipo Torsades de Pointes (TdP) constitui critério para monitoramento eletrocardiográfico a cada 8 a 12 horas após o início do tratamento. A disponibilidade de equipamentos apresenta grande variação entre as unidades de saúde. Dentre as opções disponíveis, o monitoramento automatizado constitui a melhor estratégia, seguida dos eletrodos tipo pinça eletrônicos, deixando os manuais em último lugar (DREW B, et al., 2010).

Através do monitoramento automatizado, ao detectar intervalo QT maior que $500 \mathrm{~ms}$ ou aumento de 60 ms em relação ao valor registrado antes da administração da droga, principalmente na existência de outros sinais eletrocardiográficos que sugiram uma possível TdP, tomadas de atitude são necessárias. Dentre as possíveis ações estão a mudança da estratégia farmacoterápica, a análise de possíveis interações medicamentosas agravantes e observação de bradiarritmias ou distúrbios eletrolíticos. Ainda, um desfibrilador externo deve estar prontamente disponível (DREW B, et al., 2010).

As recomendações do Ministério da Saúde em relação ao tratamento da infecção pelo SARS-Cov-2 estão fundamentadas na redução do risco dos efeitos adversos da HCQ. Ao comparar-se a dose recomendada pelo Ministério da Saúde aos estudos analisados anteriormente neste trabalho, pode-se inferir que a dose recomendada é pequena.

\section{CONSIDERAÇÕES FINAIS}

$\mathrm{CQ}$ e HCQ nas doses recomendadas pelo MS apresentam uma relação risco-benefício incerta. O risco de prolongamento do intervalo QT é relativamente alto. O benefício do uso destas drogas no tratamento da COVID-19 é discutível pela divergência dos estudos existentes, vieses consideráveis e amostras não representativas, a maioria apresentando resultados inconclusivos. A carência de pesquisas impede uma 
melhor análise da relação risco-benefício. Mais estudos são necessários, com amostras abrangentes, posologias diferentes e acompanhamentos longos. Assim poderiam ser feitas conclusões mais confiáveis sobre o uso de CQ e HCQ no tratamento da COVID-19. Reitera-se a importância da monitorização contínua do paciente em uso destas drogas para evitar os desfechos fatais do prolongamento do intervalo QT.

\section{REFERÊNCIAS}

1. BORBA MGS, et al. Chloroquine diphosphate in two different dosages as adjunctive therapy of hospitalized patients with severe respiratory syndrome in the context of coronavirus (SARS-Cov-2) infection: Preliminary safety results of a randomized, double-blinded, phase Ilb clinical trial. medRxiv, 2020.

2. CHATRE C, et al. Cardiac Complications Attributed to Chloroquine and Hydroxychloroquine: A Systematic Review of the Literature. Drug Safety, 2018; 41(10): 919-31.

3. CHEN J, et al. A pilot study of hydroxychloroquine in treatment of patients with common coronavirus disease-19 (COVID-19). J Zhejiang Univ (Med Sci), 2020; 49(1): 0-0.

4. CHEN Z, et al. Efficacy of hydroxychloroquine in patients with COVID-19: results of a randomized clinical trial. medRxiv, 2020.

5. DREW B, et al. Prevention of Torsade de Pointes in Hospital Settings: A Scientific Statement From the American Heart Association and the American College of Cardiology Foundation. Journal of the American College of Cardiology, 2010; 121: 1047-1060.

6. GAUTRET P, et al. Hydroxychloroquine and azithromycin as a treatment of COVID-19: results of an open-label nonrandomized clinical trial. International Journal of Antimicrobial Agents, 2020.

7. GELERIS J, et al. Observational Study of Hydroxychloroquine in Hospitalized Patients with Covid-19. New England Journal of Medicine, 2020.

8. GIUDICESSI JR, et al. Urgent Guidance for Navigating and Circumventing the QTc Prolonging and Torsadogenic Potential of Possible Pharmacotherapies for Covid-19. Mayo Clin Proceedings, 2020; 95.

9. HUANG M, et al. Treating COVID-19 with Chloroquine. Journal of Molecular Cell Biology, 2020; 00(00): 1-4.

10. KAPOOR A, et al. Cardiovascular risks of hydroxychloroquine in treatment and prophylaxis of COVID-19 patients: A scientific statement from the Indian Heart Rhythm Society. Indian Pacing and Electrophysiology Journal, 2020.

11. JUURLINK DN. Safety considerations with chloroquine, hydroxychloroquine and azithromycin in the management of SARS-CoV-2 infection. Canadian Medical Association Journal, 2020; 192(17): 450-453.

12. LAKKIREDDY DR, et al. Guidance for Cardiac Electrophysiology During the Coronavirus (COVID-19) Pandemic from the Heart Rhythm Society COVID-19 Task Force; Electrophysiology Section of the American College of Cardiology; and the Electrocardiography and Arrhythmias Committee of the Council on Clinical Cardiology, American Heart Association. Heart Rhythm, 2020.

13. LIU J, et al. Hydroxychloroquine, a less toxic derivative of chloroquine, is effective in inhibiting SARS-CoV-2 infection in vitro. Cell Discovery, 2020; 6(16): 1-4.

14. MINISTÉRIO DA SAỨDE. Diretrizes para diagnóstico e tratamento da COVID-19. 2020; 3: 1-79.

15. MINISTÉRIO DA SAÚDE. NOTA INFORMATIVA № 6/2020-DAF/SCTIE/MS. 2020.

16. MAHEVAS $M$, et al. No evidence of clinical efficacy of hydroxychloroquine in patients hospitalized for COVID-19 infection with oxygen requirement: results of a study using routinely collected data to emulate a target trial. medRxiv, 2020.

17. MIRANDA-AQUINO T, et al. Long QT syndrome secondary to drug interaction between hydroxychloroquine and amiodarone. Revista Mexicana de Cardiología, 2018; 29(2): 98-101.

18. PRUTKIN JM, et al. Coronavirus disease 2019 (COVID-19): Arrhythmias and conduction system disease. UpToDate, 2020.

19. SARAYANI A, et al. Safety signals for QT prolongation or Torsades de Pointes associated with azithromycin with or without chloroquine or hydroxychloroquine. Research in Social and Administrative Pharmacy, 2020.

20. TOURET F, LAMBALLERIE X. Of chloroquine and COVID-19. Antiviral Research, 2020; (177): 104762. 